\title{
CLASSIFICAÇÃO DECIMAL DE DEWEY: ALGUMAS MOTIVAÇÕES E JUSTIFICATIVAS DE USO PELA REDE DE BIBLIOTECAS DA UFMG
}

\author{
Michelle Umbelino \\ Aluna de Graduação em Biblioteconomia - ECI-UFMG \\ miumbe@gmail.com \\ Elisângela Cristina Aganette \\ Doutora em Ciência da Informação \\ Professora da Escola de Ciência da Informação, ECI-UFMG \\ DOTI - Departamento Organização e Tratamento da Informação \\ elisangelaaganette@gmail.com
}

Resumo

\begin{abstract}
Dentre as atividades desempenhadas pelos bibliotecários, a classificação se destaca. Nas bibliotecas, utiliza-se algum Sistema de Classificação Bibliográfica (SCB). Sistemas esses que permitem a definição de relações e notações entre os itens do acervo, facilitando assim a busca e a recuperação do item. Os sistemas de classificação mais utilizados mundialmente são: a Classificação Decimal de Dewey (CDD) e a Classificação Decimal Universal (CDU). Neste artigo, o SCB estudado é CDD, tem por objetivo apontar as principais razões e motivações que justifiquem o uso da CDD, como sistema de classificação padrão, e ainda identificar quais são os benefícios e possíveis dificuldades na sua utilização. Para isso analisou-se bibliotecas da Rede UFMG. Para isso, realizou-se levantamento das bibliotecas da Rede e em seguida, identificou-se quais sistemas de classificação são utilizados pelas mesmas. Aplicou-se questionários, direcionado aos bibliotecários, atuantes nas bibliotecas que utilizam ou não a CDD. Foi possível verificar que diferentemente do que se pensava, os bibliotecários que utilizam a CDD como instrumento de classificação nas bibliotecas da Rede, o fazem por acreditar que o mesmo é um tipo de sistema de classificação ideal e que atende às demandas dos leitores atendidos pela Rede. O presente estudo evidenciou a necessidade e importância da necessidade e continuidade da pesquisa no intuito de se verificar se realmente as demandas são atendidas de forma ideal, por meio da utilização da CDD, ou trata-se apenas da dificuldade de se alterar um sistema de classificação já utilizado a muito tempo.
\end{abstract}

Palavras-chave: Sistema de classificação Bibliográfico; Classificação Decimal de Dewey - CDD; Rede de Bibliotecas da UFMG.

\section{INTRODUÇÃO}

A ação de categorizar e classificar são inerentes aos seres humanos que tudo tenta categorizar e classificar desde os tempos primórdios. Foram os filósofos da antiguidade, os primeiros a se preocupar em dividir, distinguir, ou seja, em classificar os objetos materiais e formais das diversas áreas da ciência. Aristóteles teria sido o primeiro intelectual a se preocupar tanto com a questão da classificação das áreas e do saber, quanto com a organização dos documentos, naquela época pergaminhos, papiros, etc. (BARBOSA, 1969, p.15).
Ressalta-se aqui a importância de se entender que categorizar e classificar possuem diferentes significados. $\mathrm{O}$ conceito de categorizar nasceu com Platão e seu discípulo Aristóteles. "Categorias" é o primeiro dos cinco tratados que compõem a obra da lógica aristotélica "Organon", que determinou os dez gêneros supremos que constituem as Categorias: substância, quantidade, qualidade, relação, lugar, tempo, posição, posse, ação e paixão (MORA apud XAVIER, 2008). A categorização é o processo de dividir o mundo em grupos de entidades cujos membros têm similaridades entre eles dentro de um determinado contexto (JACOB, 2004). Já a 
classificação, refere-se a um processo técnico, do qual o bibliotecário é especialista. Para Ferreira (2008, p.239), classificar é "distribuir em classe e/ou grupos, segundo um sistema de classificação" e, ainda, classificação é o "[...] ordenamento de pessoas ou coisas segundo suas qualidades". Um sistema de classificação, portanto, é, de acordo com Barbosa (1969, p.23), "um conjunto de agrupamentos de assuntos coordenados e subordinados por determinadas características". A classificação é a tarefa mais importante de uma biblioteca já que constitui o meio pelo qual os documentos são utilizados. Tem por finalidade primordial a arrumação de documentos da melhor maneira para o uso e para tanto deve-se seguir um melhor método de classificação (BARBOSA, 1969).

Durante muito tempo os métodos de classificação utilizados, consideravam apenas as características físicas, tais como tamanho, cor, tipo de encadernação, etc. Assim, durante séculos, os livros eram organizados apenas com o intuito de preservá-los e não de recuperá-los. Conforme aponta Barbosa (1969), os livros eram organizados por sistemas filosóficos ou práticos. A preocupação com a classificação de acordo com o conteúdo de cada livro, passou a ser premissa, no momento em que as bibliotecas deixaram de ser simples locais para guarda e armazenamento de livros e se tornou um lugar para disponibilização, acesso e compartilhamento de informação. A partir deste momento, os bibliotecários sentiram a necessidade de uma classificação sistemática, em que os livros fossem reunidos por assunto, "a fim de melhor atender os interesses dos leitores" (BARBOSA, 1969, p.13).

$\mathrm{O}$ referido momento foi marcado por importantes pesquisas na área da classificação, uma vez que importantes e notórios estudos foram desenvolvidos, culminando em propostas de diferentes sistemas de classificação, os atuais Sistemas de Classificação Bibliográfica (SCBs). Tais sistemas buscaram facilitar o processo de classificação, por meio da unificação, em grupos, dos conhecimentos, segundo os assuntos que os abrangem, enquadrando-os num sistema pré-estabelecido que, na prática, têm a finalidade de representar, organizar, localizar, recuperar e acessar as informações.

No que se refere ao momento atual vivenciado, a prática de classificação não sofreu grande alteração, no que tange aos métodos propostos e utilizados desde os primórdios. O que mudou foi o contexto a ser classificado e o perfil do leitor que busca por informação e conhecimento. Para a classificação e definição de um modelo específico a ser seguido, primeiramente, o bibliotecário deve considerar a política de informação e respectivas diretrizes estabelecidas pela instituição.

Verifica-se que não há na Rede de Bibliotecas da UFMG, diretrizes que determine qual Sistema de Classificação Bibliográfico deve ser utilizado. Cabe a cada biblioteca definir o sistema de classificação bibliográfica mais adequado, de acordo com suas necessidades e em conformidade com os recursos humanos e tecnológicos disponíveis para devida operacionalização. Porém, percebe-se que nem sempre o SCB escolhido é a melhor opção para aquela biblioteca específica, e dessa forma, pode não atender às necessidades dos leitores. A escolha do SCB é uma decisão muito importante para a biblioteca, e deve-se pensar na aplicação, utilização e manutenção do acervo a longo prazo, uma vez que a mudança para um outro tipo de SCB demandará mais recursos financeiros, mão de obra qualificada e desconforto aos leitores.

O presente artigo teve como motivação, discussões realizadas entre as autoras deste artigo, durante a disciplina de CDD da Escola de Ciência da Informação da UFMG, no ano de 2016. Explicitado o contexto, e diante do cenário acima apresentado, o artigo buscou verificar a realidade das bibliotecas integrantes da Rede de Bibliotecas da UFMG, no que se refere aos SCB adotados para classificação de seus respectivos acervos. E ainda: i) identificar quais e quantas bibliotecas utilizam a CDD; ii) identificar razões que justifiquem a escolha pela CDD como sistema de classificação; iii) identificar possíveis dificuldades advindas especificamente pelo uso da CDD; iv) verificar se há algum tipo de limitação e ou restrição vivenciada pelos bibliotecários das referidas bibliotecas, ligadas à infraestrutura física e ou tecnológica existente; v) analisar a percepção dos bibliotecários quanto ao uso da CDD como sistema de classificação bibliográfico adotado.

Para o desenvolvimento deste estudo utilizou-se o método quantitativo e qualitativo, especificamente pelo método de perguntas, que compreende a técnica de aplicação de 
questionário e a técnica da entrevista. Para Cunha (1982), o uso de um método específico no desenvolvimento de uma pesquisa, depende dos objetivos da mesma, pois cada método apresenta tanto vantagens quanto desvantagens.

Quanto à relevância do presente estudo, trata-se de um assunto que em geral é pouco discutido por pesquisadores, e pelos profissionais que atuam nas bibliotecas da Rede. A CDD é um sistema que possui algumas limitações no que tange ao atual contexto informacional e tecnológico. Mas ainda assim, várias bibliotecas mantêm a CDD como instrumento de classificação, uma vez que não possuem recursos para realizarem tal mudança para algum outro sistema. Trata-se de uma mudança complexa, mas necessária em muitos casos. No entanto, tal necessidade ainda não é amplamente discutida pelos responsáveis das bibliotecas da Rede UFMG. Faz-se necessário conhecer a realidade das bibliotecas em termos do uso e da aplicação dos sistemas de classificação atuais. Conforme já mencionado, apesar das limitações da CDD, muitas bibliotecas da Rede ainda a utilizam. As razões pela continuidade de uso não são claras e não encontram-se documentadas na literatura da área. Assim, espera-se que o presente artigo, possa contribuir para uma melhor compreensão da realidade das bibliotecas universitárias e dos fatos que justifiquem o uso da CDD, em detrimento a outros sistemas de classificação.

\section{SISTEMAS DE CLASSIFICAÇÃO BIBLIOGRÁFICA}

Um sistema de classificação consiste em um mapa completo de qualquer área do conhecimento, mostrando todos os seus conceitos e suas relações (LANGRIDGE, 1977). No entanto, um sistema de classificação é caracterizado por Piedade (1977) como sendo um conjunto de classes apresentadas em ordem sistemática. É uma distribuição de um conjunto de ideias por certo número de conjuntos parciais, coordenados e subordinados. A autora aponta a existência de dois sistemas distintos de classificação, o filosófico e o bibliográfico: i) classificações filosóficas: buscam definir, esquematizar e hierarquizar o conhecimento, preocupados com a ordem das ciências ou a ordem das coisas; ii) classificações bibliográficas: são a base da organização de documentos $\mathrm{e}$ informações, em estantes, catálogos, bibliografias, sistemas, etc.

Barbosa (1969, p.23) considera um sistema de classificação como "um conjunto de agrupamentos de assuntos coordenados e subordinados por determinadas características", grupos estes denominados classes. As classes principais surgiram da divisão dos conhecimentos humanos em grandes grupos de acordo com algumas características oriundas dos estudos de grandes filósofos do passado. Os sistemas de classificação, na verdade, tratam de uma linguagem artificial utilizada para que os assuntos de que tratam um documento sejam trocados por sinais ou símbolos correspondentes. Estes símbolos são as chamadas notações da classificação que, junto com a notação do autor (retiradas de tabelas como a de Cutter) formam o número de chamada do documento dentro da coleção.

Os Sistemas de Classificação Bibliográfica (SCB) têm como principal função organizar acervos de bibliotecas e unidades de informação segundo os assuntos de que tratam os itens do acervo. Esses sistemas se fundamentam em três conceitos: categorias, divisão lógica e relacionamento. As classificações bibliográficas têm hoje uma utilidade indiscutível quando se trata de localizar fisicamente o documento em sua classe de assunto (AGANETTE et al 2017). Segundo Pereira et al (2009, p.2-3) a classificação bibliográfica "se preocupa com a organização e a disposição física de documentos, visando com isso, a sua recuperação. Busca ordenar, para arquivar e ter acesso ao documento em estantes ou nos arquivos".

Como exemplos podemos citar as classificações de Bliss (Bibliographic Classification), Brown (SubjectClassification), Ranganathan (ColonClassification), Dewey (Classificação Decimal de Dewey - CDD), Outlet e La Fontaine (Classificação Decimal Universal CDU), a Classificação da Library of Congress dos EUA, entre outros.

Neste artigo, o SCB que interessa é o instrumento de Classificação Decimal de Dewey (CDD), cujas características estão detalhadas na seção 2.1, a seguir. 
2.1 CLASSIFICAÇÃO DECIMAL DE DEWEY CDD

Conforme mencionado anteriormente, $\mathrm{o}$ ato de classificar é intrínseco aos processos mentais humanos e segundo Dalberg (1979), é uma arte tão antiga quanto à humanidade. A partir da classificação de Aristóteles, várias outras foram surgindo ao longo do tempo como as classificações filosóficas e bibliográficas (seção 2). Diante do atual contexto informacional e tecnológico, torna-se impossível pensar em todas as informações produzidas, e imaginar quais seriam as consequências de o ser humano não possuir mecanismos de rápido acesso às suas necessidades informacionais. Destaca-se aqui a importância de todos os sistemas de classificação existentes, em especial um tipo bibliográfico, foco do estudo: a Classificação Decimal de Dewey (CDD).

Melville Louis Kossuth Dewey (18511931), bibliotecário norte-americano e autor da CDD, desenvolveu este sistema, baseado na classificação de Harris, essência das ideias de Bacon, que via o homem como o centro do universo (LIMA, 2004). De acordo com Barbosa (1969, p. 199), Dewey, "idealizou um sistema de classificação baseado no uso dos números em ordem decimal que marcou época na história da classificação de material bibliográfico". A autora também aponta que antes de Dewey, os números na sequência decimal apenas haviam sido usados por Mame (1583) e Shurtleff (1856), como numeração de prateleiras e estantes e não como disposição dos livros entre si. A CDD utiliza números decimais de forma hierárquica para abarcar todo o conhecimento produzido. Contém as áreas fundamentais do conhecimento, do geral para o mais específico, e não apresenta um princípio explícito (LANGRIDGE, 1977).

Publicada anonimamente em 1876, a primeira edição da CDD, "Classification and Subject Índex for Cataloguing and Arranging the Books and Pamphlets of a Library", consistia em um folheto com 42 páginas divididas em 12 páginas de introdução, 12 páginas de tabelas e 18 páginas de índice. $\mathrm{O}$ famoso título "Decimal Classification and relative index" surgiu somente na $2^{\mathrm{a}}$ edição, publicada em 1885, trazendo, desta vez, indicação de responsabilidade. A partir de sua $16^{\mathrm{a}}$ edição, a obra passou então a ser denominada Dewey Decimal Classification
(DDC) ou Classificação Decimal de Dewey (CDD), traduzida para o português. Já em sua $22^{a}$ edição, continua a ser muito utilizada em bibliotecas no mundo todo. $\mathrm{O}$ que, segundo LANGRIDGE (1977), não se justifica, devido à existência de sistemas mais aprimorados, com um caráter de análise e síntese das informações.

De forma genérica e sintética pode-se dizer que a CDD é: i) um mapa completo das áreas do conhecimento, mostrando todos os seus conceitos e suas relações; ii) um sistema hierárquico, em que as ideias, os conceitos são representados em suas múltiplas relações de coordenação, de subordinação e de superordenação; iii) um sistema de classificação decimal que adota como princípio fundamental a divisibilidade do todo em dez partes, baseando-se numa divisão inicial em disciplinas e subdisciplinas; iv) um sistema de classificação estruturado, primordialmente bibliográfico, destinado a servir de base à organização de documentos; v) um sistema de classificação enumerativo, relaciona todos os assuntos $\mathrm{e}$ todas as combinações/associações/relações possíveis entre os mesmos, juntamente com seus símbolos/combinações de símbolos para consumo, sem (maiores) intervenções do classificador (SILVA E GANIN, 1994).

Quanto a sua estrutura, a CDD é composta por dez classes, denominadas de Main Classes, e que Dewey dividiu nove destas para representar o conhecimento humano, e destacou a necessidade de uma décima classe onde pudessem reunir obras que tratassem sobre todos os assuntos. As main classes são:

000 Computer science, information \& general works

100 Philosophy \& psychology

200 Religion

300 Social sciences

400 Language

500 Science

600 Technology

700 Arts \& recreation

800 Literature

\section{History \& geography}

Cada uma dessas classes possui outras nove subdivisões, e cada umas destas subdivisões 
possui mais nove seções, contabilizando ao todo dez classes, cem subdivisões e mil seções. A CDD conta com algumas tabelas auxiliares, que como o próprio nome indica, possibilitam maior detalhamento do assunto a ser classificado:

Tabela 1: Standart Subdivisions

Tabela 2: Geographic areas, historical periods, persons

primários e da realização de uma pesquisa empírica, que utilizou como Etapa instrumento de coleta, entrevistas.

Realizaram-se as seguintes etapas, detalhadas a seguir:

i) Levantamento preliminar das bibliotecas da Rede UFMG.

a. Por meio do acesso ao site da Rede (https://www.bu.ufmg.br/bu/),

Tabela 3: Subdivisions for individual literatures for specific lifelizagufsems $\mathrm{um}$ levantamento de Tabela 4: Subdivisions for individual languages

Tabela 5: Racial, Ethnic, national groups

Tabela 6: Languages

Tabela 7: Group of persons.

A CDD apresenta ainda, um índice ordenado alfabeticamente, conhecido como índice relativo, ou seja, indica sob cada assunto todos os pontos do sistema em que se encontram seus vários aspectos, incluindo também entradas relativas aos termos das tabelas auxiliares.

A CDD, atualmente, segundo Andrade, Bruna e Sales (2011, p.35), "é o sistema de classificação bibliográfica mais utilizado em todo o mundo". Segundo os autores, embora não esteja divulgada nos meios de comunicação, a CDD "é sem dúvida um dos mais importantes inventos da humanidade", pois, o que seria da humanidade, caso não houvesse uma forma estruturada de se acessar de forma rápida o que se precisa de informação? Os autores consideram ainda, que a CDD se destaca dos demais sistemas de classificação bibliográfica por possuir "uma maior autonomia no aspecto do idioma" já que foi pensado para o uso na cultura norte americana. A contribuição de Dewey para a biblioteconomia é indiscutível. Tais contribuições somadas às contribuições de Ranganathan vislumbram caminhos que levem ao efetivo acesso à informação (LIMA NETO, 2011).

\section{METODOLOGIA}

A proposta geral do estudo consiste em verificar a realidade das bibliotecas integrantes da Rede de Bibliotecas da UFMG, no que se refere aos SCB adotados para classificação de seus respectivos acervos. Para isso utilizou-se o método qualitativo e quantitativo de pesquisa, por meio da coleta de dados quantas e quais são as bibliotecas que fazem parte do Sistema de Bibliotecas da UFMG.

b. Criação de uma lista com a relação de todas as bibliotecas e seus respectivos responsáveis (bibliotecário ou outro profissional).

c. Envio de e-mail para cada um dos responsáveis (bibliotecário ou outro profissional). Explicou-se o objetivo do contato e formalizou-se o convite para realização da entrevista online ou presencial, de acordo com a disponibilidade do entrevistado.

ii) Etapa dois: Elaboração do questionário para entrevista dos responsáveis (bibliotecário ou outro profissional) por cada uma das bibliotecas, independente do sistema de classificação utilizado.

iii) Etapa três:

Levantamento do sistema de classificação utilizado em cada biblioteca da Rede.

Envio do questionário, APÊNDICE A, via e-mail para todas as bibliotecas da Rede. Em alguns casos a entrevista ocorreu de forma presencial, de acordo com a disponibilidade do entrevistado;

a. Análise dos questionários respondidos;

Criação da lista com a relação das bibliotecas que utilizam a CDD.

iv) Etapa quatro: Elaboração do questionário, APÊNDICE B, para entrevista dos responsáveis (bibliotecário ou outro profissional) por cada uma das bibliotecas que utilizam a CDD.

v) Etapa cinco: Realização de 
entrevistas com responsáveis (bibliotecário ou outro profissional) por cada uma das bibliotecas que utilizam a CDD.

vi) \begin{tabular}{llr} 
Etapa seis: & \multicolumn{2}{c}{ Análise } \\
consolidação & dos & dados \\
levantados & durantes & as
\end{tabular} levantados
entrevistas.

\section{RESULTADOS E DISCUSSÃO}

De acordo com o levantamento realizado o Sistema de Bibliotecas da UFMG é composto, atualmente, por 26 bibliotecas. Essas bibliotecas estão subordinadas, administrativamente, às Unidades Acadêmicas, Escolas de Educação Básica e Profissional e outros órgãos suplementares. Conforme o relatório anual do Sistema de Bibliotecas (2015), o Sistema de Bibliotecas da UFMG, em 2015, contava com um acervo impresso de 1.032 .460 exemplares, uma coleção impressa de 15.256 periódicos e ainda 43.623 itens no acervo de materiais especiais, referentes à diversas áreas do conhecimento. $\mathrm{O}$
Sistema, contava com 184.993 usuários inscritos e realizou 682.288 empréstimos domiciliares. As bibliotecas que fazem parte do Sistema de Bibliotecas da UFMG, e seus respectivos SCB adotados, estão dispostos no Quadro 1.

Ressalta-se que, as etapas de levantamento, não foram tarefas fáceis de se cumprir. Cabe dizer que o momento histórico vivenciado pela comunidade da UFMG não estava propicio para tal levantamento. No final do segundo semestre de 2016, muitos servidores aderiram à greve, e várias unidades da Universidade encontravam-se "ocupadas" pelos alunos, em forma de protesto. Tal momento atrasou a evolução do estudo e, somente com a retomada do semestre, no início de 2017, pode-se retomar a pesquisa, ou seja, reenviar os e-mails e assim realizar novas entrevistas, para se chegar aos resultados apresentados a seguir. Evidencia-se, por meio do Quadro 1, que de um total de 26 bibliotecas pertencentes à Rede de Bibliotecas da UFMG, 12 utilizam a CDD, 12 a CDU, 01 utiliza a Classificação de Black e 01 utiliza o NLM como sistema de classificação bibliográfico padrão.

Quadro 1: Panorama da Rede de Bibliotecas da UFMG, quanto aos SCB utilizados

\begin{tabular}{|c|c|c|}
\hline & Biblioteca & Sistema de Classificação \\
\hline 1 & Biblioteca Central & $\mathrm{CDU}^{1}$ \\
\hline 2 & Biblioteca da Escola de Arquitetura & $\mathrm{CDD}^{2}$ \\
\hline 3 & Biblioteca da Escola de Belas Artes & CDD \\
\hline 4 & Biblioteca da Escola de Ciência da Informação & $\mathrm{CDU}$ \\
\hline 5 & Carro-biblioteca - Projeto de Extensão & CDD \\
\hline 6 & $\begin{array}{l}\text { Biblioteca da Escola de Educação Física, Fisioterapia e Terapia } \\
\text { Ocupacional }\end{array}$ & $\mathrm{CDU}$ \\
\hline 7 & Biblioteca da Escola de Engenharia & $\mathrm{CDU}$ \\
\hline 8 & Biblioteca da Escola de Música & CDD \\
\hline 9 & Biblioteca da Escola de Veterinária & CDD \\
\hline 10 & $\begin{array}{l}\text { Biblioteca do Centro Pedagógico da Escola de Educação Básica } \\
\text { e Profissional da UFMG }\end{array}$ & $\mathrm{CDD}$ \\
\hline 11 & Biblioteca da Faculdade de Ciências Econômicas & CDD \\
\hline 12 & Biblioteca da Faculdade de Direito & $\mathrm{CDU}$ \\
\hline 13 & Biblioteca da Faculdade de Educação & CDD \\
\hline 14 & Biblioteca da Faculdade de Farmácia & CDD \\
\hline 15 & Biblioteca da Faculdade de Filosofia e Ciências Humanas & CDD \\
\hline 16 & Biblioteca da Faculdade de Letras & CDD \\
\hline 17 & Biblioteca da Faculdade de Odontologia & CDD/Classif. de Black ${ }^{3}$ \\
\hline 18 & Biblioteca do Campus Saúde & $\mathrm{NLM}^{4}$ \\
\hline 19 & $\begin{array}{l}\text { Biblioteca do Colégio Técnico da Escola de Educação Básica e } \\
\text { Profissional da UFMG }\end{array}$ & $\mathrm{CDU}$ \\
\hline
\end{tabular}

${ }^{1}$ Classificação Decimal Universal

${ }^{2}$ Classificação Decimal de Dewey

${ }^{3}$ Classificação de Black, classificação específica para a área da odontologia.

${ }^{4}$ National Library of Medicine, classificação específica para a área da medicina. 
Relatos de Pesquisa

\begin{tabular}{|l|l|l|}
\hline \hline 20 & Biblioteca do Departamento de Física & CDU \\
\hline 21 & Biblioteca do Departamento de Química & CDU \\
\hline 22 & $\begin{array}{l}\text { Biblioteca do Instituto de Ciências Biológicas (incorporada à } \\
\text { Biblioteca Central) }\end{array}$ & CDU \\
\hline 23 & Biblioteca do Instituto de Ciências Exatas & CDU \\
\hline 24 & Biblioteca do Instituto de Geociências & CDU \\
\hline 25 & Biblioteca do Museu de História Natural & CDU \\
\hline 26 & Biblioteca do Instituto de Ciências Agrárias & CDU \\
\hline
\end{tabular}

Fonte: Dos autores

Após realizada a primeira etapa da pesquisa, que se consolidou nos dados apresentados no Quadro 1, deu-se início a segunda etapa. Etapa esta que teve como foco a realização de novas entrevistas. No entanto, conforme mencionado na metodologia, nesta etapa, apenas os responsáveis por cada uma das 12 bibliotecas da Rede que utilizam a CDD como sistema de classificação foram novamente entrevistados, conforme APÊNDICE B. Sendo elas:

Biblioteca da Escola de Arquitetura Biblioteca da Escola de Belas Artes

Carro-biblioteca - Projeto de Extensão

Biblioteca da Escola de Música

Biblioteca da Escola de Veterinária

Biblioteca do Centro Pedagógico da Escola de

Educação Básica e Profissional

Biblioteca da Faculdade de Ciências Econômicas

Biblioteca da Faculdade de Educação

Biblioteca da Faculdade de Farmácia

Biblioteca da Faculdade de Filosofia e Ciências

Humanas

Biblioteca da Faculdade de Letras

Biblioteca da Faculdade de Odontologia

O referido questionário foi enviado por email aos responsáveis de cada uma das 12 bibliotecas da Rede, no entanto não obteve-se um retorno de $100 \%$ dos responsáveis, uma vez que apenas 10 entrevistados deram o devido retorno, o que em percentual equivale a $83 \%$ dos responsáveis pelas bibliotecas da Rede. Todos os 10 questionários foram respondidos por bibliotecários com vasta experiência, variando entre 6 e 26 anos de atuação como bibliotecário. Ressalta-se que dos 10 profissionais entrevistados, 3 sempre trabalharam com a CDD, sendo assim desconhecem a aplicação de outro tipo de $\mathrm{SCB}$, apenas 1 bibliotecário posicionou-se e evidenciou sua insatisfação com a CDD, afirmando que a mesma não é adequada para seu contexto, outros 3 bibliotecários se posicionaram de forma positiva no que se refere ao uso da $\mathrm{CDD}$, afirmando não encontrar dificuldades na utilização da mesma. Outros 3 entrevistados, apontam o idioma da publicação como um dificultador; e ainda: a subjetividade na interpretação dos assuntos pelos profissionais; a localização do assunto, dentro de determinada área do conhecimento; a ausência de classes mais específicas, tendo que se classificar os itens de forma geral; e a dificuldade de se utilizar as tabelas auxiliares para se especificar um assunto. Os 10 bibliotecários entrevistados nesta segunda etapa, afirmaram que os problemas advindos da aplicação da CDD não estão ligados à infraestrutura física, tecnológica ou às suas próprias condições de trabalho.

Para melhor apresentar as dificuldades apontadas, destacamos duas respostas do questionário:

\begin{abstract}
Nos primeiros contatos com a CDD e também quando surge a necessidade de criar uma notação muito específica com utilização das tabelas e exclusão de algum dígito no momento de unir ou relacionar dois ou mais assuntos, tendo a preocupação de não criar uma notação muito grande e que possa dificultar a busca e a organização do item na estante, visto que nosso foco vai do público infantil ao adulto (Entrevistado 1).
\end{abstract}

Localização do assunto, dentro de determinada área do conhecimento. Outro aspecto deve-se ao surgimento de novas áreas, nem sempre a edição da CDD que a Biblioteca possui acompanha essa atualização. Um exemplo: área do Design. (O bibliotecário precisa de tempo para desenvolver algumas classificações especificas que são tratadas de forma geral na CDD) (Entrevistado 7).

Quanto às vantagens relacionadas ao uso da CDD, obteve-se o seguinte resultado: 6 entrevistados apontam a notação simplificada como a principal vantagem; 1 entrevistado evidencia a questão da padronização e do compartilhamento, por acreditar que a maioria 
das bibliotecas da UFMG utilizam a CDD, auxiliando assim na definição do número de classificação. Apesar de o entrevistado pontuar que a maioria das bibliotecas da Rede utilizam a CDD, por meio do presente estudo, evidenciou-se que a quantidade de bibliotecas que utilizam a CDD como SCB, é a mesma das que utilizam a CDU, ou seja, 12 bibliotecas usam CDD e 12 a CDU. O que reafirma o que já foi mencionado no presente artigo, pouca discussão no que tange a utilização prática dos SCBs.

Para melhor evidenciar as vantagens apontadas, destacam-se as respostas de dois entrevistados:

Apesar de ser em inglês, a composição do número de classificação é mais simples para o usuário. A CDD possui uma abrangência maior, o que facilita na hora de classificar. Pelo fato de aqui na UFMG a maioria das bibliotecas utilizarem a CDD também ajuda quando vamos definir o número de classificação (Entrevistado 8).

Apesar das dificuldades, como o idioma da obra, a CDD permite atribuir número de classificação mais objetivo (menos complexo) (Entrevistado 5).

Apenas um entrevistado, utilizou o campo "observação" no questionário, para o seguinte posicionamento:

Com relação ao arquivamento nas estantes, particularmente acho que a notação da CDD é mais fácil na hora da guarda dos itens, enquanto a CDU torna-se mais complicada por utilizar símbolos e sinais juntamente com números, mais nada que um bom treinamento não possa resolver o problema (Entrevistado 1).

Diante das análises dos questionários e das conversas presenciais, percebe-se que na maioria das vezes a prevalência e manutenção do uso da CDD nas bibliotecas, está relacionada ao fato de já se utilizar tal SCB a muito tempo, ou seja, a velha premissa do "sempre foi feito assim", "sempre utilizou a CDD”, e não ao melhor SCB para atendimento às demandas de uma biblioteca, à busca de um SCB ideal.

\section{CONSIDERAÇÕES FINAIS}

Pode-se dizer que não existe um modo ideal de se classificar e organizar o mundo de forma a atender às expectativas de todos os usuários de informação. Entretanto, tudo tem o seu devido lugar conforme a necessidade e o contexto. Por isso é importante que se utilize algum tipo de SCB de acordo com as necessidades e especificidades do contexto informacional a ser classificado, uma vez que a maneira que a informação retornará ao usuário será relativa ao contexto buscado por ele.

Diante do objetivo geral do presente artigo, de se conhecer a realidade das bibliotecas pertencentes a Rede de Bibliotecas da UFMG, no que tange ao tipo de SCB adotado, especificamente ao contexto das bibliotecas que adotam a CDD como SCB padrão para classificação de seus acervos, percebe-se que as principais limitações e dificuldades, apontadas pelos bibliotecários, estão no idioma o qual a obra está disponível e no próprio ato subjetivo de se classificar algo, uma vez que envolve a interpretação pessoal do bibliotecário.

Por meio do presente artigo, foi possível formalizar o quadro atual de bibliotecas da Rede UFMG, no que se refere a quantas e quais utilizam a CDD ou outro tipo de SCB. Sendo que das 26 bibliotecas pertencentes à Rede, 12 utilizam a CDD, 12 utilizam a CDU e duas outros tipos. No início da pesquisa acreditavase que o número de bibliotecas que utilizam a CDD fosse bem menor que o resultado obtido. Não encontrou-se nenhum documento oficial informando qual tipo de SCB é utilizado pelas bibliotecas da Rede da UFMG.

Percebe-se que os bibliotecários não vinculam os problemas ou as limitações (restrições) enfrentadas no dia a dia em suas unidades de informação, pelo uso da CDD a questões de infraestrutura física $\mathrm{e}$ ou tecnológica, ou a outras condições de trabalho. Consideram a CDD um sistema simples e que atende à demanda da biblioteca, apresentando poucas dificuldades na sua utilização. Ou seja, os bibliotecários consultados não consideram a CDD um sistema limitado, e segundo os mesmos, a mantem como SCB padrão, uma vez que a consideram apropriada às demandas da unidade e não pelo fato de não possuir recursos para realizar tal mudança, seja para a CDU ou para outro SCB mais completo. Ressalta-se que como trabalho futuro, complementar ao presente artigo, será necessário, para verificar se realmente as 
demandas das bibliotecas são atendidas pelo uso da CDD, realizar novas entrevistas, agora obrigatoriamente presencial, com os bibliotecários que responderam aos questionários, nesta fase da pesquisa. Isso se justifica, pelo fato do momento conturbado vivenciado pela comunidade acadêmica da UFMG. Percebeu-se que os entrevistados, por disporem de pouco tempo para se dedicar ao questionário, se limitaram a respostas curtas, $\mathrm{o}$ que pode ter prejudicado o resultado e consequentemente as análises das autoras

\section{DEWEY DECIMAL CLASSIFICATION: MOTIVES AND JUSTIFICATIONS OF APPLICATION IN THE UFMG LIBRARY NETWORK}

\section{Abstract}

Among the activities carried out by librarians, the classification stands out. In libraries, some Bibliographic Classification System (SCB) is used. These systems allow the definition of relationships and notations among the items in the collection, thus facilitating the search and retrieval of the item. The most widely used classification systems in the world are: the Dewey Decimal Classification (CDD) and the Universal Decimal Classification (CDU). In this article, the SCB studied is $C D D$, it aims to identify the main reasons and motivations that justify the use of CDD, as a standard classification system, and to identify the benefits and possible difficulties in its use. For this, UFMG Network libraries were analyzed. For this, a survey of the network libraries was carried out and then, it was identified which classification systems are used by them. Questionnaires were sent to librarians working in libraries that use CDD or not. It was possible to verify that, differently from what was thought, the librarians who use the CDD as a classification tool in the libraries of the Network do so because they believe that it is a type of ideal classification system that meets the demands of the readers served by the Network. The present study evidenced the necessity and importance of the necessity and continuity of the research in order to verify if the demands are actually met ideally through the use of CDD, or it is only the difficulty of changing a classification system already used for a long time.

Keywords: Bibliographic classification system; Dewey Decimal Classification - DDC; UFMG Library Network.

Artigo recebido em: 18/09/2017

Aceitação definitiva em: 10/12/2017

\section{REFERÊNCIAS}

AGANETTE, Elisângela Cristina et al. Ações para o Processo de Ensino Aprendizagem em Disciplinas de Organização e Tratamento da Informação: inovação pedagógica no grupo de pesquisa RECRI/UFMG. ISKO Portugal Espanha, 2017.

ANDRADE, Lucas Veras de; BRUNA, Dayane; SALES, Weslayne Nunes de. Classificação: Uma análise comparativa entre a Classificação Decimal Universal - CDU e a
Classificação Decimal de Dewey - CDD.

Biblos: Revista do Instituto de Ciências

Humanas e da Informação, v. 25, n.2, p.3142, jul./dez. 2011. Disponível em:

<https://www.seer.furg.br/biblos/article/view/ 2088>. Acesso em: 10 jul. 2017.

BARBOSA, Alice Príncipe. Teoria e prática dos sistemas de classificação bibliográfica. Rio de Janeiro: IBBD, 1969. 441p. CUNHA, Murilo Bastos da. Metodologias para estudo dos usuários de Informação cientifica e tecnológica. Revista de Biblioteconomia de Brasília, v.10, n.2, p.5- 
19. jul./dez. 1982. Disponível em: <http://bogliolo.eci.ufmg.br/downloads/CUN HA_1982.pdf>. Acesso em: 15 out. 2016.

DAHLBERG, Ingetraut. Teoria da classificação, ontem e hoje. In: CONFERÊNCIA BRASILEIRA DE CLASSIFICAÇÃO BIBLIOGRÁFICA, 1976, Rio de Janeiro. Anais... Rio de Janeiro: IBICT; Brasília: ABDF, 1979. p. 352-370.

FERREIRA, Aurélio Buarque de Holanda. Mini Aurélio: o dicionário da língua portuguesa. $7^{\mathrm{a}}$ ed. Curitiba: Positivo, 2008, 896p.

JACOB, Elin. Classification and categorization: a difference that makes a differance. Library trends, v. 52, n. 3, p. 515-540, 2004. Disponível em:

$<$ https://pdfs.semanticscholar.org/774e/ab27b 22aa92dfaa9aeeeafbe845058e85f58.pdf $>$. Acesso em: 10 jul. 2017.

LANGRIDGE, Derek. Classificação: abordagem para estudantes de biblioteconomia. Rio de Janeiro: Interciência, 1977.

LIMA NETO, José Domingos de.

Classificação bibliográfica e o uso das cores como aspecto facilitador no acesso à informação em bibliotecas. 2011. 62f. TCC (Graduação em Biblioteconomia) - Centro de artes e comunicação. Universidade Federal De Pernambuco, Recife, 2011. Disponível em: <http://www.liber.ufpe.br/bibtcc/files/p/397/3 97.pdf>. Acesso em: 10 jul. 2017.

LIMA, Vânia Mara Alves. Da classificação do conhecimento científico aos sistemas de recuperação de informação: enunciação de codificação e enunciação de decodificação da informação documentária. 2004. 117f. Tese (Doutorado em Ciência da Comunicação) Universidade de São Paulo, São Paulo, 2004.

PEREIRA, Edinete do Nascimento et al. Classificação bibliográfica: as diversas contribuições para o tratamento da informação. In: SEMINÁRIO DE PESQUISA DO CENTRO DE CIÊNCIAS SOCIAIS APLICADAS, 15.; 2009, Natal, RN. Anais... Universidade e os Desafios da Formação na Sociedade do Conhecimento. Natal, R. 2009. 12p. Disponível em: <https://repositorio.ufrn.br/jspui/bitstream/1/6 173/1/(Classifica\%C3\%A7\%C3\%A3o\%20bib $\operatorname{liogr} \% \mathrm{C} 3 \% \mathrm{~A} 1$ fica).pdf $>$. Acesso em: 15 out. 2016.

PIEDADE, Maria Antonietta Requião. Introdução à teoria da classificação. Rio de Janeiro: Interciência, 1977. 185p.

SILVA, Odilon Pereira da, GANIM, Fátima. Manual da CDU. Brasília: Briquet de Lemos/Livros, 1994. 89p.

XAVIER, Beatriz Rêgo. As categorias de Aristóteles e o conhecimento científico. Pensar, Fortaleza, v. 13, n. 1, p. 57-64, jan./jun. 2008. 


\section{APÊNDICE A - Questionário utilizado em todas as bibliotecas da Rede}

O presente questionário faz parte de um estudo para a disciplina de Sistemas de Classificação Bibliográfica, do curso de Biblioteconomia que tem como objetivo fazer um levantamento de quais sistemas de Classificação Bibliográfica são utilizados pelas bibliotecas da Universidade Federal de Minas Gerais. Os dados levantados serão utilizados para o trabalho de conclusão da disciplina. Sua colaboração no preenchimento do mesmo será de grande importância para a conclusão do mesmo.

1- Nome da Biblioteca:

2- Localização:

3- Nome do(s) responsável(is) pela catalogação do acervo:

4- Contatos (e-mail e telefone):

5- Qual sistema de classificação bibliográfica utilizado na biblioteca?
( ) CDD
( ) $\mathrm{CDU}$
( ) Outro. Qual?

6- Estaria disposto a participar de um estudo sobre a prática na utilização do sistema de classificação bibliográfica na biblioteca em que você trabalha?
( ) SIM
( ) NÃO
( ) TALVEZ 


\section{APÊNDICE B - Questionário utilizado nas bibliotecas da Rede que utilizam a CDD}

O presente questionário faz parte de um estudo para a disciplina de Sistemas de Classificação Bibliográfica, do curso de Biblioteconomia que tem como objetivo fazer um levantamento de quais sistemas de Classificação Bibliográfica são utilizados pelas bibliotecas da Universidade Federal de Minas Gerais, identificar se existem dificuldades no trabalho de catalogação utilizando a CDD e se existem, quais são estas dificuldades; identificar se as limitações (restrições) que os bibliotecários enfrentam no dia a dia nessas unidades de informação, estão ligadas à infraestrutura física e tecnológica e às condições de trabalho e analisar a percepção dos bibliotecários sobre o uso do sistema de classificação bibliográfica. Os dados levantados serão utilizados para o trabalho de conclusão da disciplina. Sua colaboração no preenchimento do mesmo será de grande importância para a conclusão do mesmo.

1. Qual o seu nome?

2. Qual a sua formação?

3. Quais as suas funções?

4. A quanto tempo atua nestas funções?

5. Você conhece ou já trabalhou com outros sistemas de classificação? ( ) SIM ( ) NÃO Se sim quais?

6. Consegue apontar diferenças entre os sistemas de seu conhecimento?

7. Você tem ou já teve dificuldades para utilizar a CDD? ( ) SIM （ ) NÃO

8. Se sim, quais as principais dificuldades?

9. Você acredita que essas dificuldades, estão ligadas à infraestrutura física, tecnológica e às suas condições de trabalho? ( ) SIM

10. Se sim, aponte o que você acredita ser a causa principal:

11. Você acredita que a CDD é o melhor sistema de classificação para sua biblioteca?

$$
\text { ( ) SIM ( ) NÃO }
$$

12. Se sim, aponte as principais vantagens na sua utilização.

13. Se não, qual seria o melhor sistema, na sua opinião?

14. Alguma observação que queira acrescentar que não destacamos neste questionário? 